\title{
Taking the Next Steps in Endoscopic Visual Assessment of Barrett's Esophagus: A Pilot Study
}

\author{
Roxana Chis $\mathbb{D}^{\prime}$ \\ Simon $\mathrm{Hew}^{2}$ \\ Wilma Hopman ${ }^{3}$ \\ Lawrence Hookey ${ }^{2}$ \\ Robert Bechara (D) ${ }^{2}$ \\ 'Division of Internal Medicine, Kingston \\ Health Sciences Centre, Queen's \\ University, Kingston, Ontario, Canada; \\ ${ }^{2}$ Division of Gastroenterology, Kingston \\ Health Sciences Centre, Queen's \\ University, Kingston General Hospital, \\ Kingston, Ontario, Canada; ${ }^{3}$ Research \\ Institute, Kingston Health Sciences \\ Centre, Queen's University, Kingston, \\ Ontario, Canada
}

Correspondence: Robert Bechara Division of Gastroenterology, Queens University, Kingston Health Sciences Center, 166 Brock Street, Kingston,

Ontario K7LIA8, Canada

Tel+ 6135443400

Email bechara.robert@gmail.com
Purpose: Patients with Barrett's esophagus (BE) undergo surveillance endoscopies to assess for pre-cancerous changes. We developed a simple endoscopic classification method for predicting non-dysplastic BE (NDBE), low-grade dysplasia (LGD)/indefinite for dysplasia (ID) and high-grade dysplasia (HGD)/early esophageal adenocarcinoma (EAC).

Patients and Methods: Twenty-two patients with BE underwent endoscopy using the PENTAX Medical MagniView gastroscope and OPTIVISTA processor. Sixty-six video-still images were analyzed to characterize the microsurface, microvasculature and the presence of a demarcation line. Class A was characterized by regular microvascular and microsurface patterns and absence of a demarcation line, class B by changes in the microvascular and/or microsurface patterns compared to the background mucosa with presence of a demarcation line, and class $\mathrm{C}$ by irregular microvascular and/or irregular microsurface patterns with presence of a demarcation line.

Results: Of the class A images, 97.9\% were NDBE. For class B, 69.2\% were LGD/ID and $30.8 \%$ NDBE. One hundred percent of the class C samples were HGD/EAC. The sensitivity of our classification system was $93.8 \%$, specificity $92 \%$, positive predictive value $78.9 \%$, negative predictive value $97.9 \%$ and an accuracy $92.4 \%$.

Conclusion: In this study, we developed a simple classification system for the prediction of NDBE, LGD/ID and HGD/EAC. Its real-time clinical applicability will be validated prospectively.

Keywords: Barrett's esophagus, BE, dysplasia, enhanced endoscopy, esophageal adenocarcinoma

\section{Introduction}

Esophageal adenocarcinoma poses a significant disease burden across the world, affecting 52,000 people annually. ${ }^{1}$ Furthermore, the prognosis for esophageal adenocarcinoma is poor, with an overall 5-year survival rate of $17 \%$ and is significantly lower in advanced diseases. ${ }^{2}$

Esophageal adenocarcinoma, however, has a precursor lesion, Barrett's esophagus (BE). Barrett's esophagus is a condition consisting of metaplastic changes to the lining of the distal esophagus as a result of epithelial injury and inflammation from chronic gastroesophageal reflux and it has the potential to gradually progress to esophageal carcinoma. ${ }^{3}$ The progression to esophageal adenocarcinoma is thought to occur in sequence from non-dysplastic Barrett's esophagus (NDBE) to low-grade dysplasia (LGD) to high-grade dysplasia (HGD) and finally progression to esophageal adenocarcinoma (EAC). ${ }^{4,5}$ Patients with BE are at increased risk of developing EAC, with an annual risk of adenocarcinoma of $0.12 \%{ }^{6}$ 
Due to this risk of esophageal cancer, it is recommended that patients with $\mathrm{BE}$ undergo regular surveillance endoscopies with biopsies to assess for any pre-cancerous changes. ${ }^{7}$ Prescriptive biopsies, however, even following the Seattle protocol, can only sample a low percentage of affected mucosa. There is therefore a risk of missing concerning dysplastic or even neoplastic lesions.

An alternative surveillance strategy is the use of targeted biopsies. Advancements in endoscopic technology now offer the ability to potentially detect neoplastic esophageal lesions through enhanced visualization of the esophageal mucosa. ${ }^{8}$ These technologies include special staining of the esophageal mucosa with chromoendoscopy, virtual chromoendoscopy, confocal endomicroscopy, endocytoscopy, autofluorescence or optical coherence tomography. ${ }^{9}$

Chromoendoscopy, virtual chromoendoscopy, and confocal laser endomicroscopy are the most widely studied techniques. Chromoendoscopy utilizes dyes to improve visualization of the esophageal mucosa and to enhance the mucosal patterns associated with dysplasia; virtual chromoendoscopy, including narrow band imaging, uses light filters within the endoscope to highlight vessel and mucosal patterns; confocal laser endomicroscopy magnifies the gross image of the esophageal mucosa by up to thousand-fold. ${ }^{10}$ These advanced imaging techniques can significantly increase the diagnostic yield for identification of dysplasia or adenocarcinoma in patients with BE. ${ }^{10}$ However, none of these systems have been widely adopted, due to their complexity, cost and variable diagnostic yield. ${ }^{11}$ Therefore, a void exists for a simple, userfriendly approach to identify and classify dysplastic and neoplastic lesions in BE.

We aimed to develop a simple and easily applicable endoscopic classification for the prediction of nondysplastic BE (NDBE), low-grade dysplasia (LGD)/ Indefinite for dysplasia (ID) and high-grade dysplasia (HGD)/early esophageal adenocarcinoma (EAC) utilizing a commercially available magnifying endoscope and processor.

\section{Patients and Methods}

\section{Patients}

Patients, aged 18 and older, with known or suspected BE were invited to participate in the study. Exclusion criteria included patients with prior esophageal interventions, such as dilations, stenting, radiofrequency ablation, and/or endoscopic mucosal resection, due to the effects these may have on the appearance of the esophageal mucosa. The study was conducted at the Kingston Health Sciences Center, Kingston, Ontario, Canada. The protocol for this study was approved by the ethics committee at Queen's University School of Medicine. The study protocol followed the ethical guidelines of the 1975 Declaration of Helsinki. Written informed consent was obtained from all the patients.

\section{Endoscopy}

Patients received topical anesthetic with lidocaine spray for the oropharynx and received conscious sedation as per institutional protocol with intravenous midazolam and fentanyl. One experienced therapeutic endoscopist (R.B.) completed all the gastroscopies. The PENTAX Medical MagniView gastroscope (Pentax i10 series) and OPTIVISTA processor (Pentax Corp., Tokyo, Japan) were used for all studies. The OPTIVISTA processor uses i-SCAN Optical Enhancement (OE) technology with filters to highlight features of the microsurface. OE mode 1 delivers wavelengths of 415 and $540 \mathrm{~nm}$ to highlight the microvasculature. After the usual exam of the stomach and duodenum, the esophagus was washed of any mucous with the use of simethicone and $\mathrm{N}$-acetylcysteine. The extent of BE was defined according to the Prague $\mathrm{C}$ and $\mathrm{M}$ criteria. The mucosa was optically examined for any signs suggestive of dysplasia (nodularity, demarcation lines, changes or irregularity in the microsurface or microvasculature). This was initially done with white light endoscopy without magnification, followed by $\mathrm{OE}$ mode 1 with and without magnification (up to 135x). Four quadrant biopsies were obtained every $2 \mathrm{~cm}$ for each patient as per the Seattle protocol. The number of biopsies depended on the length of the BE segment. In addition, targeted biopsies that were labelled individually were also taken and processed into separate blocks. Using standard biopsy forceps, biopsies were obtained from the abnormal areas identified. All biopsies were read by a tertiary-center gastrointestinal pathologist. Any biopsy with dysplasia or neoplasia was reviewed by a second pathologist.

Videos of the exams were recorded. Still images with magnification of the biopsied areas were obtained from the video recording and manually analyzed by an expert endoscopist. These images were used to develop our classification system. Patients found to have dysplasia were managed as per standard of care, with endoscopic resection [either mucosal resection (EMR) or endoscopic submucosal dissection (ESD)] with subsequent ablative therapy for the remaining NDBE. 


\section{Classification of Surface and Vascular Pattern}

We focused on three characteristics: microvascular pattern (regular, irregular or absent), microsurface pattern (regular, irregular or absent) and the presence of a demarcation line to create our classification system. The classification consists of three categories, aiming to distinguish between NDBE, LGD/ID and HGD/EAC. Regular microsurface patterns included regular repeating patterns consisting of oval, round, linear, curved and branched patterns of the marginal crypt epithelium. A change in microsurface patterns was marked by a different microsurface as compared to the background $\mathrm{BE}$ mucosa. Irregular microsurface patterns were characterized by breaks in the marginal crypt epithelium, varying widths, and irregular patterns in the marginal crypt epithelium. Regular microvascular patterns were regular repeating coiled, branched, reticular or honeycomb patterns and irregular microvascular patterns were described as having varying caliber, shape, branches and morphology or absent microvasculature. The presence of a demarcation line between different surface patterns was also noted and included in the classification system (Figure 1). Islands with no clear demarcation line were omitted in the analysis.

Based on differences in vascular pattern, surface pattern and demarcation line, we created a classification system (Table 1) that consists of three classes:

1. Class A: regular microvascular pattern and regular surface pattern without a demarcation line (Figure 1A)

2. Class B: a change in the microvascular pattern and/or microsurface pattern as compared to the background Barrett's mucosa with a demarcation line (Figure 1B)

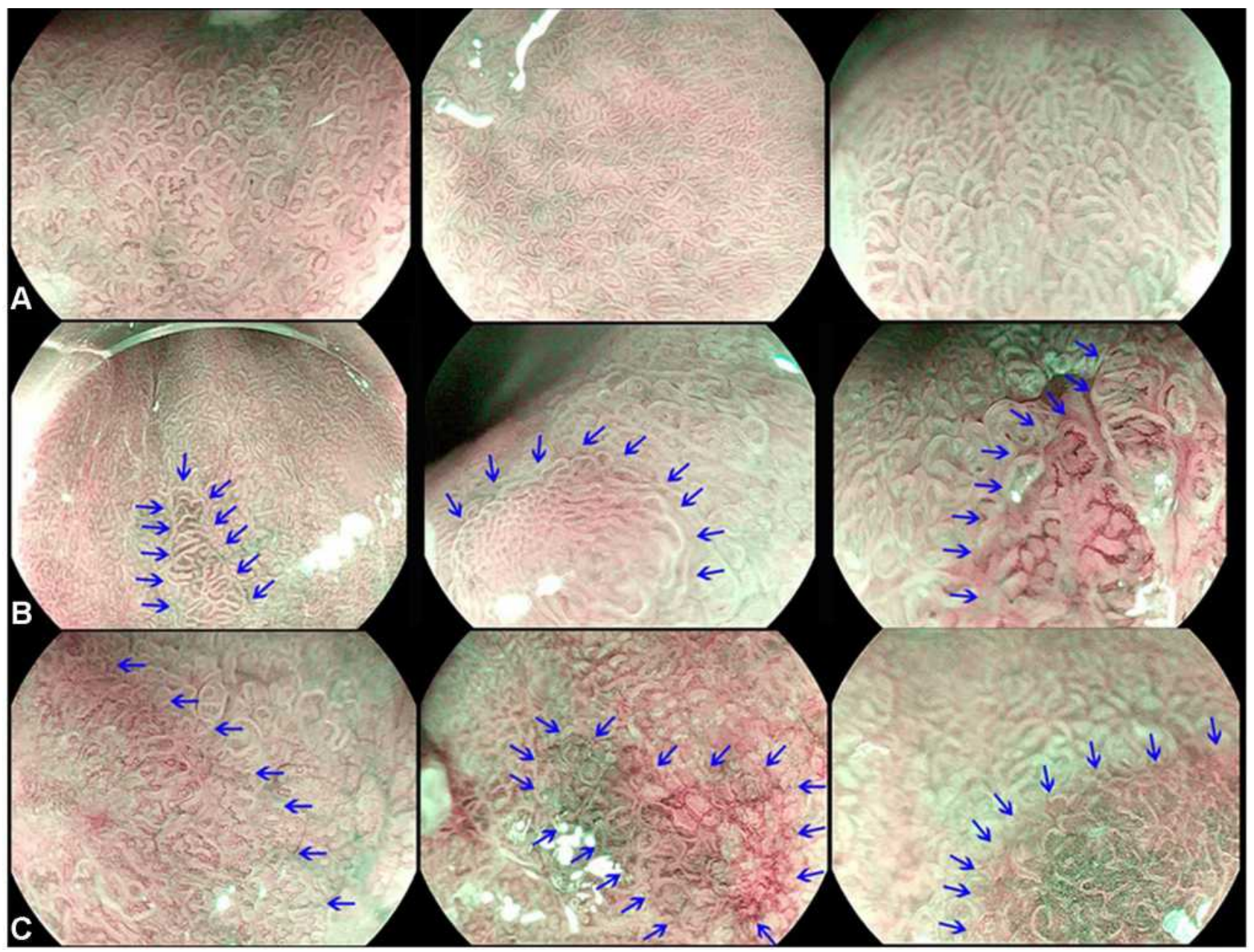

Figure I Magnified endoscopic view of esophageal mucosa of (A) Regular mucosal pattern and regular vessels that follow the architecture of the mucosa. (B) Change in the microsurface and/or microvasculature from background mucosa with demarcation marked by blue arrows. (C) Irregular microsurface and/or microvascular patterns with demarcation marked by blue arrows. 
Table I Classification System Based on Microvasculature, Microsurface and Demarcation Line Patterns

\begin{tabular}{|l|l|}
\hline Class A & $\begin{array}{l}\text { I. No microvascular irregularity } \\
\text { 2. No microsurface irregularity } \\
\text { 3. No demarcation line }\end{array}$ \\
\hline Class B & $\begin{array}{l}\text { I. Change in microvascular pattern } \\
\text { 2. Change in microsurface pattern } \\
\text { 3. Demarcation line }\end{array}$ \\
\hline Class C & $\begin{array}{l}\text { I. Irregular microvascular pattern } \\
\text { 2. Irregular microsurface pattern } \\
\text { 3. Demarcation line }\end{array}$ \\
\hline
\end{tabular}

3. Class C: irregular microvascular pattern and/or irregular microsurface pattern with a demarcation line (Figure 1C).

\section{Statistical Analysis}

The relationship between the different mucosal and vascular patterns and histology-confirmed dysplastic grade was tested. The association between the classification system derived from still images and the different histologic dysplastic categories on biopsies from the same patients was then tested. All the suspicious targeted areas were biopsied and the corresponding still images were used to calculate the positive predictive value, negative predictive value and accuracy of our classification system.

\section{Results}

Twenty-two patients undergoing surveillance or endoscopic treatment for BE were enrolled in the study (Figure 2). The patients' mean age was 64.1 years (range $48-89$ years) and $72.7 \%$ were male. Using the Prague criteria, the mean circumferential extent of BE was $2.9 \mathrm{~cm}$ (range $0-15 \mathrm{~cm}$ ) and the mean maximal extent of BE was $6 \mathrm{~cm}$ (range 1-15 cm). Fifty percent of the patients had a hiatus hernia with a mean size of $2 \mathrm{~cm}$ (Table 2).

Sixty-six biopsies paired with 66 still images were included in the analysis. Of the biopsies, 50 (75.8\%) were NDBE, 10 (15.2\%) LGD/ID and 6 (9\%) HGD/EAC. One patient with a clearly invasive carcinoma was excluded from

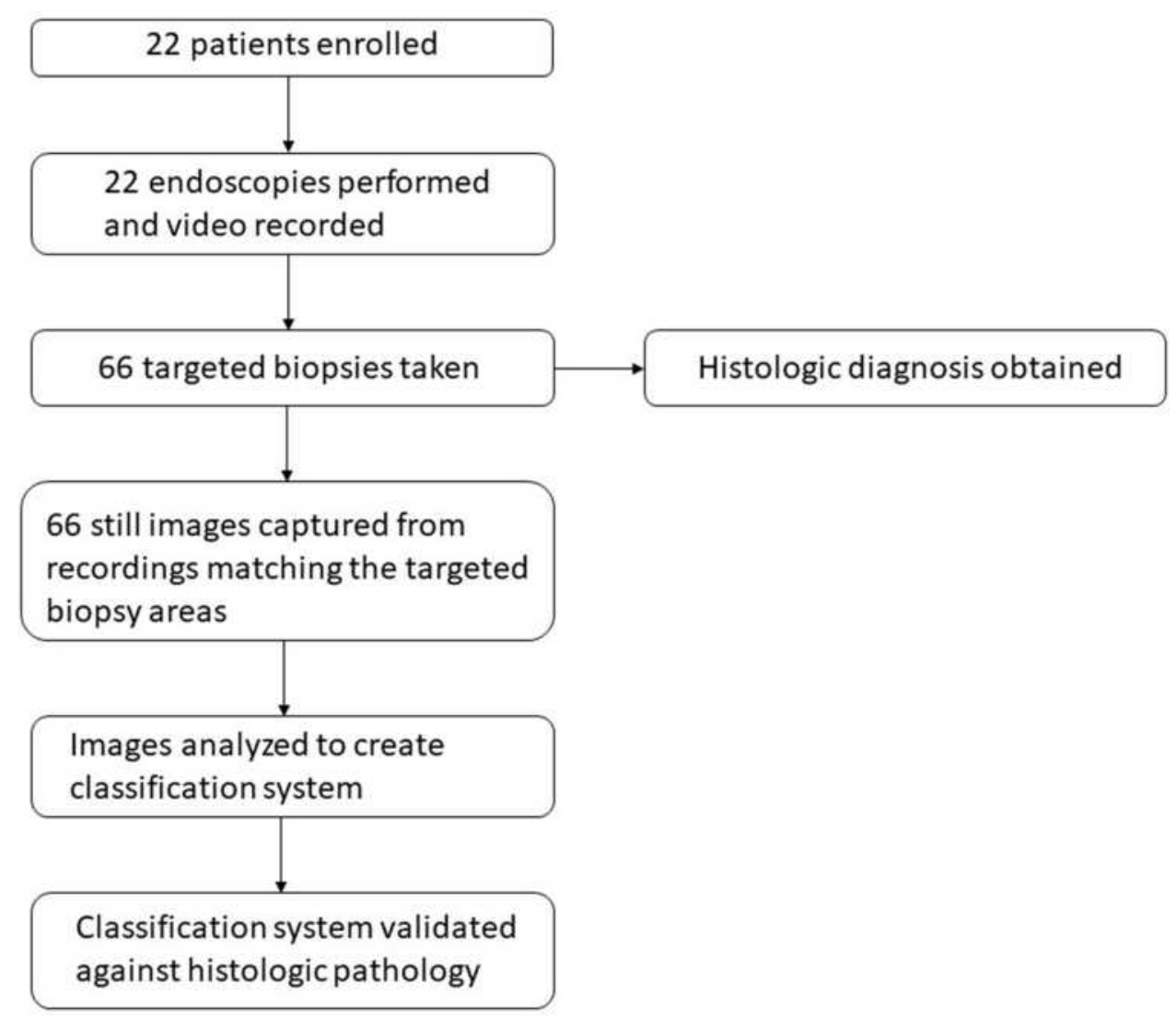

Figure 2 Study design. Twenty-two patients with known or suspected BE were enrolled in the study and underwent endoscopy. The endoscopies were video-recorded and still shots were obtained which were then used to devise the classification system. The classification system was then blindly tested against the histologic findings from targeted biopsies for its predictive capacity. 
Table 2 Clinical Characteristics of Patients Undergoing Assessment

\begin{tabular}{|l|l|}
\hline Total number of patients & 22 \\
\hline Mean age, y (range) & 64.1 (48-89) \\
\hline Sex, no. male/female & $16 / 6(72.7 / 27.3 \%)$ \\
\hline Mean circumferential BE length, cm (range) & $2.9(0-15)$ \\
\hline Hiatal hernia, no. & $11(50 \%)$ \\
\hline Histology results, percent & \\
NDBE & $50(75.8 \%)$ \\
LGD/indefinite & $10(15.2 \%)$ \\
HDG & $6(9 \%)$ \\
\hline Highest final pathologic diagnosis, no. & $13(59 \%)$ \\
NDBE & $4(18 \%)$ \\
LGD/ID & $5(23 \%)$ \\
HGD/EAC & \\
\hline
\end{tabular}

the analysis. A range of pathology was noted in certain patients based on targeted biopsies taken. Three patients showed variability in pathology with areas of NDBE and LGD. One patient showed variability with areas of NDBE and EAC, another patient had areas of LGD and indefinite for dysplasia and one patient showed variability in pathology with areas of NDBE, LGD and HGD.

When analyzing the images for vascular pattern, 49 of 66 samples were thought to have a regular vascular pattern. Of these, 47 images (95.9\%) with a regular microvascular pattern were found to be NDBE and 2 images (4.1\%) were LGD. If there was a change in the microvascular pattern as compared to the background mucosa, 8 images (72.7\%) were LGD/ID and 3 images $(27.3 \%)$ were NDBE. If there was an irregular microvascular pattern, 6 images (100\%) correlated with HGD/EAC (Figure 3).

Analysis of the microsurface pattern demonstrated that 50 samples had a regular surface pattern. Of the images with a regular microsurface, 47 (94\%) were NDBE and 3 images $(6 \%)$ were LGD. If there was a change in the microsurface pattern as compared to the background mucosa, $6(66.7 \%)$ of the images were found to be LGD/ID and $3(33.3 \%)$ were NDBE. If there was an irregular microsurface pattern, 6 images (100\%) were HGD/EAC (Figure 4).

Regarding a demarcation line of the 50 NDBE samples included in the analysis, 46 (92\%) did not have a demarcation line. The 4 (8\%) samples that did have a demarcation line were not dysplastic, however they contained inflammatory changes. In contrast, most of the dysplastic samples had a demarcation line. Of the $8 \mathrm{LGD} /$ ID, $7(87.5 \%)$ had a demarcation line and in $100 \%$ of the 6 HGD/EAC had a demarcation line (Figure 5).

Combining all three characteristics into our classification system, of the class A images, 46 images (97.9\%) were NDBE, 1 image (2.1\%) was LGD/ID and none were HGD/

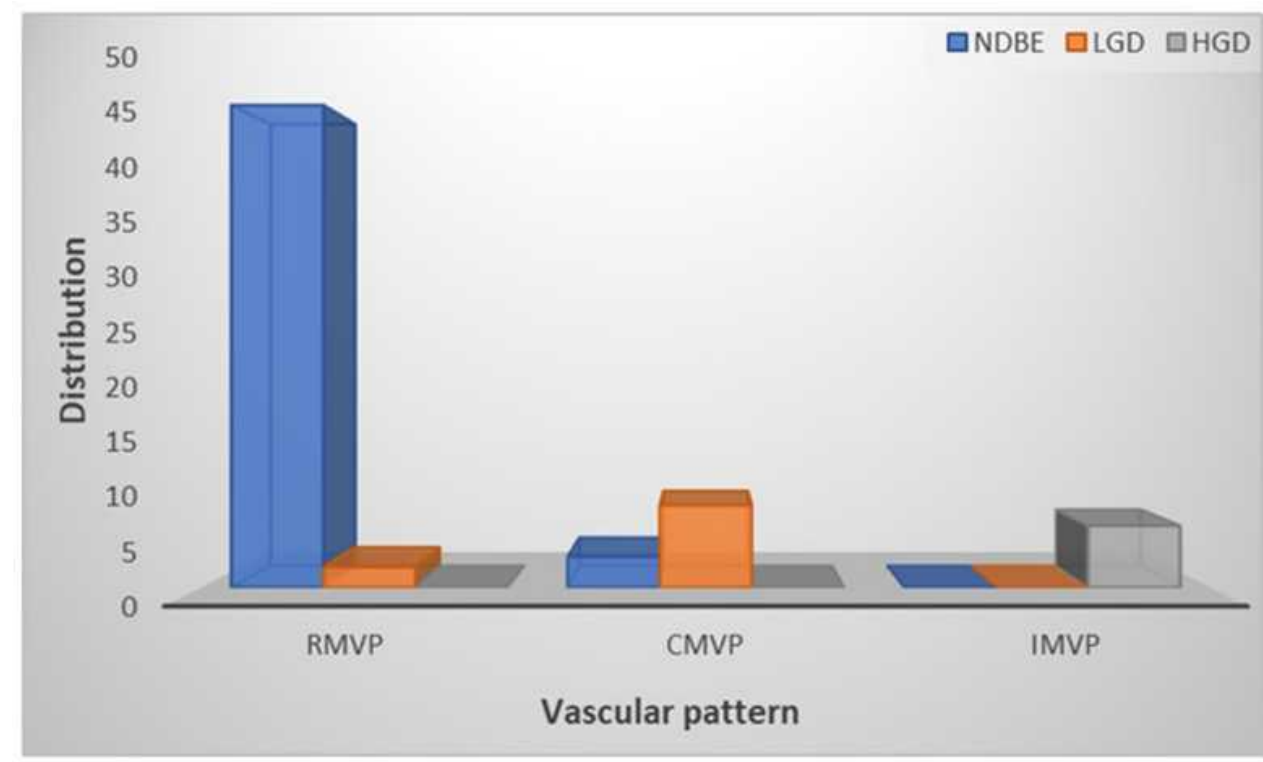

Figure 3 Distribution of dysplastic categories based on vascular pattern. The majority of samples with a RMVP were NDBE (47 of 49 ) or $95.9 \%$ compared to 2 or $4.1 \%$ LGD and no HGD. Eleven samples were thought to have a CMVP as compared to the background mucosa. Of these, 8 or $72.7 \%$ were LGD compared to 3 or $27.3 \%$ NDBE and no HGD. Six samples were found to have an IMVP and I00\% of the images correlated with HGD.

Abbreviations: RMVP, regular microvascular pattern; CMVP, change in microvascular pattern; IMVP, irregular microvascular pattern. 


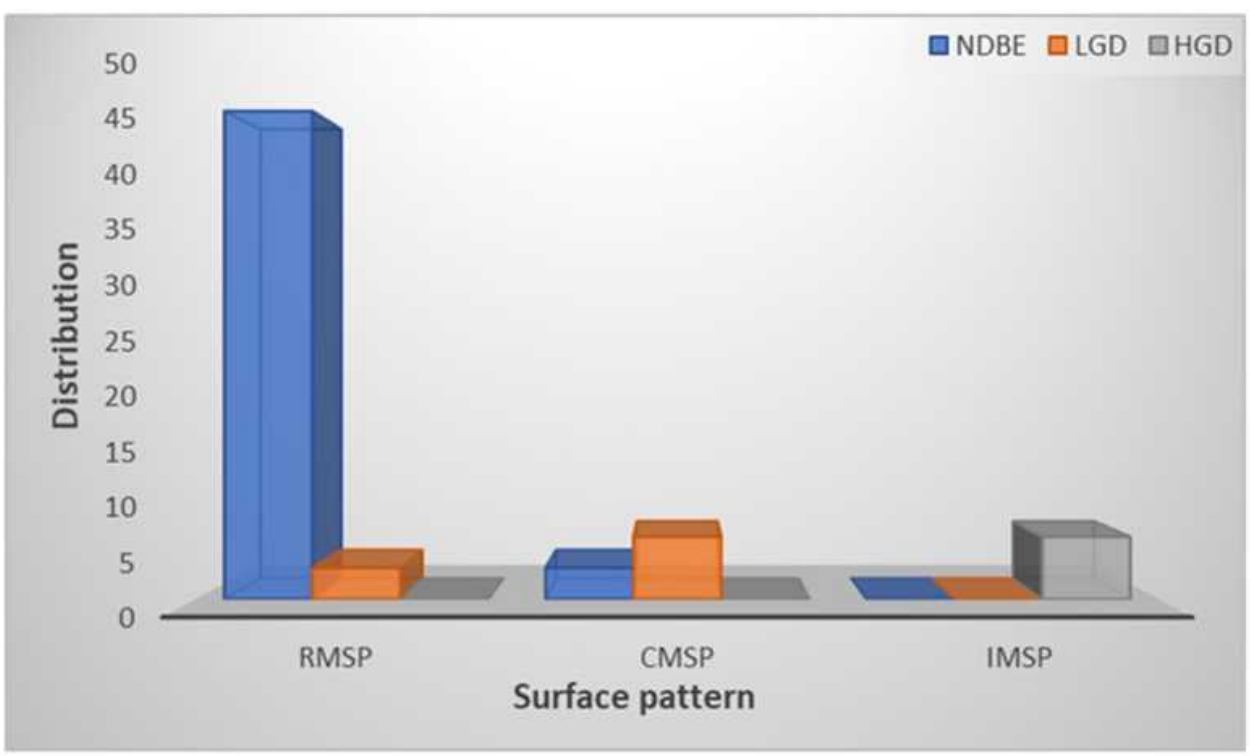

Figure 4 Distribution of dysplastic categories based on surface pattern. Of the samples with a RMSP, 47 of 50 or $94 \%$ were NDBE compared to $6 \%$ LGD and no HGD. Six of nine samples with a CMSP or $66.7 \%$ were found to be LGD compared to 3 or $33.3 \%$ NDBE and no HGD. If there was an IMVP, I00\% of the 6 images were HGD.

Abbreviations: RMSP, regular microsurface pattern; CMSP, change in microsurface pattern; IMSP, irregular microsurface pattern.

EAC. For class B, 9 images (69.2\%) were LGD/ID, 4 images were $(30.8 \%) \mathrm{NDBE}$ and none were HGD/EAC. All 6 (100\%) class C images were HGD/EAC (Figure 6). For predicting neoplasia (LGD/ID/HGD/EAC) in our cohort, our classification had a sensitivity of $93.8 \%$, specificity of $92 \%$, positive predictive value of $78.9 \%$, a negative predictive value of $97.9 \%$ and an accuracy of $92.4 \%$.

\section{Discussion}

In this pilot study, using magnification and optical enhancement technology, we developed a simple, and clinically applicable classification system for the prediction of NDBE, LGD/ID and HGD/EAC. Using three elements, a demarcation line and changes in microvascular and microsurface patterns, our classification system had a sensitivity

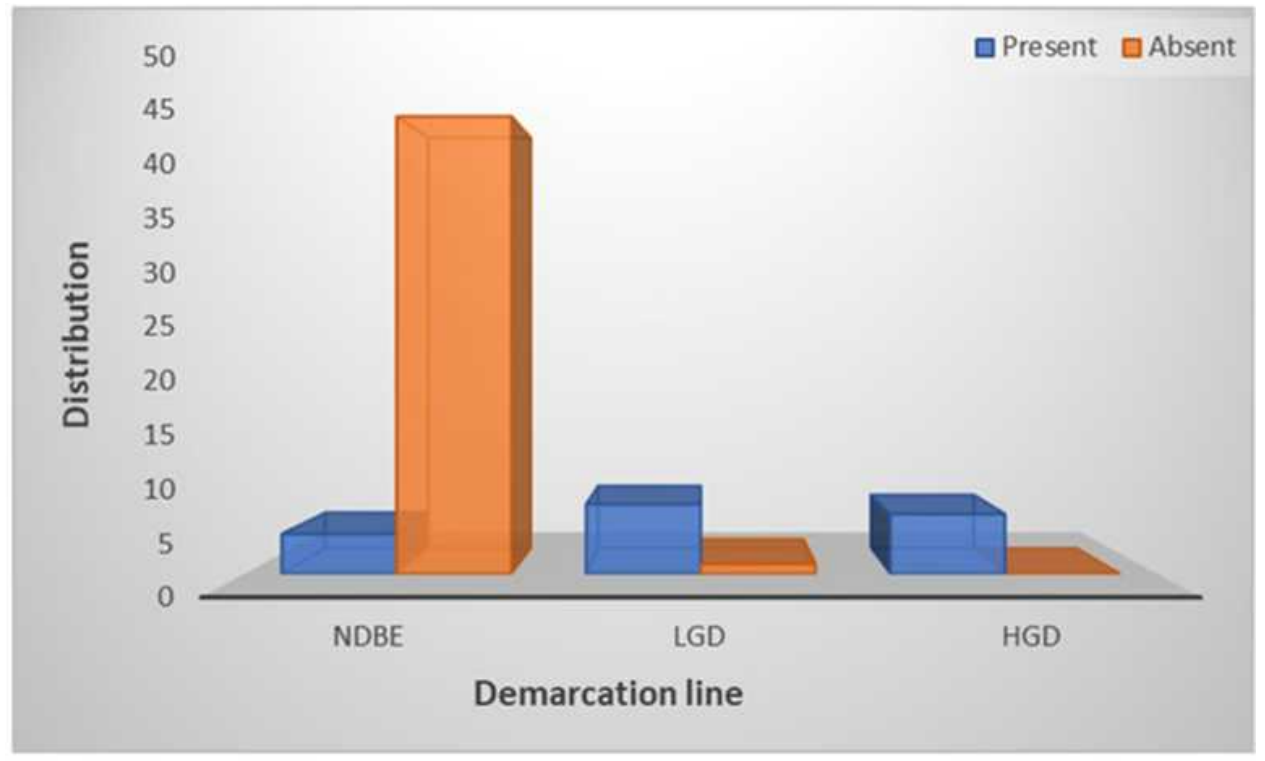

Figure 5 Distribution of dysplastic categories based on demarcation line. Regarding a demarcation line 46 of 50 or $92 \%$ of the samples did not have a demarcation line and only 4 or $8 \%$ did. In contrast, 7 of 8 LGD samples or $87.5 \%$ and $100 \%$ of the 6 HGD samples had a demarcation line.

Abbreviations: NDBE, non-dysplastic Barrett's esophagus; LGD, low-grade dysplasia; HGD, high-grade dysplasia. 


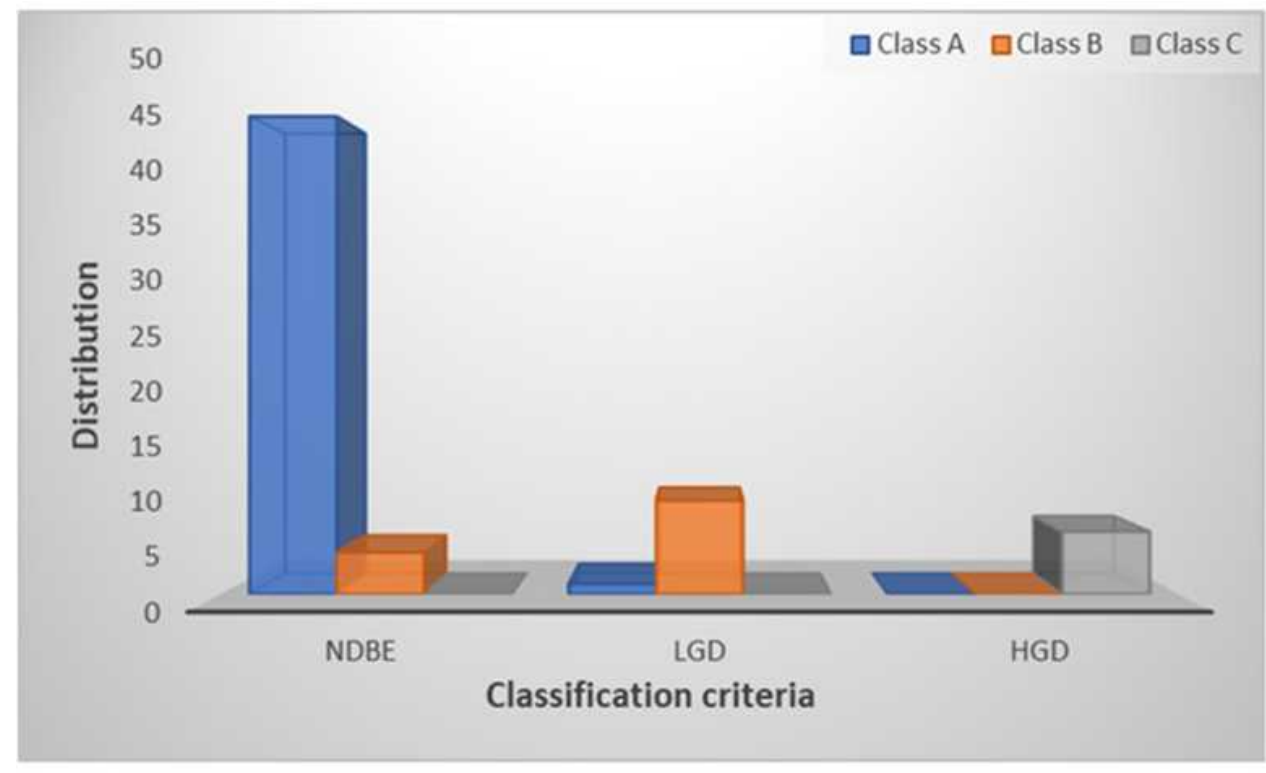

Figure 6 Pathology distribution by class. Class A pathology was predictive of NDBE in 46 of 47 or $97.9 \%$ of cases, class B predicted LGD in 9 of I3 or $69.2 \%$ of cases and class $C$ predicted HGD in $100 \%$ of 6 cases.

Abbreviations: NDBE, non-dysplastic Barrett's esophagus; LGD, low-grade dysplasia; HGD, high-grade dysplasia.

of $93.8 \%$, specificity of $92 \%$, positive predictive value of $78.9 \%$, a negative predictive value of $97.9 \%$ and an accuracy of $92.4 \%$ for predicting neoplasia (LGD/ID/HGD/EAC).

$\mathrm{BE}$ is a pre-malignant esophageal condition that has the potential to progress to cancer through a proposed metaplasia-dysplasia-carcinoma sequence. ${ }^{12}$ Therefore, the goal of endoscopic surveillance in patients with $\mathrm{BE}$ is the detection of dysplastic changes amenable to endoscopic treatment. ${ }^{12}$ The argument for endoscopic surveillance is that it detects neoplastic progression earlier than in the general population leading to improved survival rates. ${ }^{13}$ There are challenges with current surveillance protocols and certain lesions can be missed. For example, a meta-analysis of resection studies found that $39.9 \%$ of patients who underwent esophageal resection for HGD had either intramucosal carcinoma or invasive carcinoma. ${ }^{14}$ Due to the limitations of white light endoscopy, efforts have been made to enhance visualization with advanced imaging techniques. Advanced imaging techniques have been found to increase the diagnostic yield for detection of dysplasia and cancer. A meta-analysis and systematic review found improved detection of dysplasia and cancer by $34 \%$ when using advanced imaging techniques such as chromoendoscopy and virtual chromoendoscopy compared to white light endoscopy and random biopsies. ${ }^{10}$ Furthermore, techniques such as narrow band imaging have been shown to have advantages over white light endoscopy, by allowing endoscopists to obtain target rather than random biopsies and therefore reducing the number of biopsies required for processing and histopathological analysis. ${ }^{15}$ Table 3 summarizes advanced endoscopy techniques and their clinical application and performance characteristics. ${ }^{16-18}$

Multiple classification systems exist, using vascular and surface patterns, for the prediction of histopathology based on endoscopic narrow band imaging focused. ${ }^{19-21}$ For instance, the Amsterdam classification is focused on regular versus irregular vascular and mucosa patterns and normal versus abnormal vessels and it has good diagnostic value with a sensitivity of $94 \%$, a specificity of $76 \%$, a positive predictive value (PPV) of $64 \%$, and a negative predictive value (NPV) of $98 \%$ for high grade intraepithelial neoplasm. ${ }^{20}$ The Kansas classification focuses on 3 mucosal and 3 vascular patterns to distinguish between BE and HGD and its sensitivity, specificity and PPV of irregular/distorted pattern for HGD were 100\%, 98.7\%, and $95.3 \%$, respectively. ${ }^{19}$ The Nottingham group focused on 4 mucosal and 4 vascular classifications to distinguish between columnar mucosa, BE and HGD with $81 \%$ and 99\% PPV and NPV, respectively, for detection of HGD. ${ }^{22}$ More recently, a simplified classification system was developed by the BING consortium focusing on regularity versus irregularity of mucosal and vascular patterns and the group reported an accuracy of $85 \%$, sensitivity of $80 \%$, 
Table 3 Overview of Advanced Endoscopic Imaging Techniques

\begin{tabular}{|c|c|c|}
\hline Technique & Description & Comments \\
\hline Chromoendoscopy & $\begin{array}{l}\text { Chromoendoscopy involves the application of dyes or chemicals } \\
\text { (methylene blue, indigo carmine, and acetic acid) onto the mucosa } \\
\text { to enhance the visualization of mucosal and microvascular } \\
\text { patterns. }\end{array}$ & $\begin{array}{l}\text { Used in identification of Barrett's esophagus by } \\
\text { enhancing the differences between metaplastic and non- } \\
\text { metaplastic mucosa. } \\
\text { Challenges include lack of standardization of technique. } \\
\text { Depending on staining method: } \\
\text { Sensitivity } 64-96 \% \\
\text { NPV } 69-98 \% \\
\text { Specificity } 84-99 \%\end{array}$ \\
\hline $\begin{array}{l}\text { Narrow Band } \\
\text { Imaging (NBI) }\end{array}$ & $\begin{array}{l}\text { NBI uses narrow-band optical filters to improve the vascular } \\
\text { contrast of capillaries and submucosal vessels. }\end{array}$ & $\begin{array}{l}\text { Used in the identification of Barrett's esophagus by } \\
\text { revealing vascular and mucosal patterns. } \\
\text { Easy to use, but problems with pattern recognition and } \\
\text { steep learning curve. } \\
\text { Sensitivity } 94 \% \text {, NPV } 97 \% \text {, Specificity } 94 \%\end{array}$ \\
\hline i-SCAN & $\begin{array}{l}\text { i-SCAN uses post-processing algorithm to enhance image } \\
\text { contrast based on reflective differences of normal and abnormal } \\
\text { mucosa. }\end{array}$ & $\begin{array}{l}\text { Structure and vascular enhancement. } \\
\text { More data needed for clinical applicability. }\end{array}$ \\
\hline $\begin{array}{l}\text { Confocal Laser } \\
\text { Endomicroscopy } \\
\text { (CLE) }\end{array}$ & $\begin{array}{l}\text { CLE allows for in vivo microscopic level magnification of the } \\
\text { epithelium. }\end{array}$ & $\begin{array}{l}\text { Identification of Barrett's glands and cells. Allows for } \\
\text { real-time viewing of cellular details. } \\
\text { Extensive training required for diagnosis accuracy. } \\
\text { Sensitivity } 90 \% \text {, NPV } 98 \% \text {, Specificity } 92 \%\end{array}$ \\
\hline $\begin{array}{l}\text { Artificial } \\
\text { Intelligence (Al) }\end{array}$ & Computer-aided diagnosis of Barrett's esophagus. & $\begin{array}{l}\text { Machine learning algorithms that can analyze texture } \\
\text { and colour in order to predict dysplastic vs non- } \\
\text { dysplastic lesions. } \\
\text { Sensitivity } 83-95 \% \text {, Specificity } 0.85-100 \% \\
\text { Accuracy } 89-92 \%\end{array}$ \\
\hline
\end{tabular}

specificity of $88 \%$, PPV of $81 \%$, and NPV of $88 \%$ for detection of dysplasia. ${ }^{23}$ However, these classification systems have only satisfactory inter-observer agreement on further validation, i.e. there were no significant differences in dysplasia detection between the different classification systems or between experienced and inexperienced assessors. $^{24,25}$ Furthermore, all these classifications have been dichotomous and are only able to distinguish between NDBE and any neoplasia.

In this pilot study, using PENTAX Medical MagniView gastroscope and OPTIVISTA processor capable of optical enhancement, we developed a new classification system based on differences in vascular and surface patterns and the presence or absence of a demarcation line. These three variables were specifically chosen for our classification system as they have proven to be very useful in the detection of early gastric cancer. ${ }^{26}$ In gastric cancer, three microvascular/microsurface patterns were described - regular, irregular and absent - and according to these patterns, endoscopists could differentiate neoplastic from non-neoplastic lesions. ${ }^{27}$ For instance, the criteria for diagnosing gastric cancer based on the presence of irregular microvascular and microsurface patterns and the presence of a demarcation line and early gastric cancer fit these criteria in $97 \%$ of cases. ${ }^{28}$

The strength of our proposed classification is that it has the potential to classify mucosa in a spectrum including NDBE, LGD/ID and HGD/EAC. The morphology of the intestinal metaplasia in $\mathrm{BE}$ is not always uniform and therefore areas of heterogeneity can exist even in the nondysplastic morphology. The intestinal metaplasia of $\mathrm{BE}$ can be heterogeneous and resemble the mucosa of the gastric antrum/body, and as such there can be a demarcation between these morphologies. This is one of the reasons why BE endoscopic classifications are challenging. In addition, the inter-observer variability between pathologists for $\mathrm{LGD} / \mathrm{ID}$ is quite variable, $\mathrm{k}=0.60 .{ }^{29}$ The heterogeneity of the class of LGD/ID is captured by the 
heterogeneity in class B lesions. A further strength of the study is the review of the biopsies by a tertiary-center gastrointestinal pathologist with a review of dysplasia on the spectrum (NDBE, LGD/ID, HGD, EAC). We do acknowledge the significant inter-observer variability in the diagnosis of dysplasia in Barrett's esophagus even among expert gastrointestinal pathologists, with $\mathrm{k}$ values of 0.22 for NDBE, 0.11 for LGD and 0.43 for HGD cited previously in literature ${ }^{30}$ possibly introducing uncertainty in the performance metrics of the endoscopic classification system when tested against highly variable histologic diagnoses. However, despite sub-optimal agreement in the histopathologic diagnosis on $\mathrm{BE}$, histologic interpretation by an expert gastrointestinal pathologist remains the gold standard in the diagnosis of BE dysplasia and therefore the most appropriate benchmark against which to assess endoscopic classification systems. While in this study, the evaluation of the still images was made by an experienced endoscopist, another strength of our system lies with the fact that its elements are very user-friendly and systematic. By following the systematic steps, any endoscopist can obtain targeted biopsies of suspicious areas thus leading to improved diagnostics.

Should our classification be validated prospectively and meet the Preservation and Incorporation of Valuable Endoscopic Innovation thresholds, ${ }^{31}$ a targeted biopsy/ resection system could be implemented; class A pattern would require no biopsy, class B pattern would lead to a biopsy and class $\mathrm{C}$ pattern would lead to resection.

Our study is limited by its small sample size and single operator performance. Another limitation is the absence of a massively invasive cancer category. Invasive carcinoma, however, is expected to be evident in endoscopy and classifying findings based on microvascular and microsurface regularity may not add additional benefit. Devising the classification system is also more time-consuming than the minimum $1 \mathrm{~min} / \mathrm{cm}$ endoscopic evaluation standard. Despite this, it can be performed in real time and within a time frame consistent with generally accepted endoscopy booking times.

Furthermore, our findings require prospective validation among multiple endoscopists. Future studies from our group will focus on validating this classification system prospectively in a larger cohort by multiple endoscopists in real time. The endoscopic classification in this study was also performed by analyzing still images. Future directions for our group will include predicting the level of dysplasia in real time and validating it against histologic diagnosis. Overall, the goal of our classification system is to ensure that abnormal areas of $\mathrm{BE}$ are detected and sampled. Further studies could focus on extending our classification system to an automated AI image analysis model.

\section{Conclusion}

In summary, using magnification and optical enhancement technology, we developed a simple, and clinically applicable classification system for the prediction of NDBE, LGD/ID and HGD/EAC. We hope that with prospective validation this classification system will be a valuable tool in the endoscopic assessment of Barrett's esophagus.

\section{Acknowledgments}

The authors would like to thank Pentax Medical for funding this project.

\section{Disclosure}

Dr Robert Bechara reports grants from Pentax Medical, during the conduct of the study; Consultant for Olympus and Pentax, outside the submitted work. The authors report no other conflicts of interest in this work.

\section{References}

1. Arnold M, Soerjomataram I, Ferlay J, Forman D. Global incidence of oesophageal cancer by histological subtype in 2012. Gut. 2015;64 (3):381-387. doi:10.1136/gutjnl-2014-308124

2. Rustgi AK, El-Serag HB, Ingelfinger JR. Esophageal carcinoma. $N$ Engl $J$ Med. 2014;371(26):2499-2509. doi:10.1056/ NEJMra1314530

3. Spechler S. Barrett esophagus and risk of esophageal cancer: a clinical review. JAMA. 2013;310(6):627-636. doi:10.1001/jama.2013.226450

4. Evans JA, Early DS, Fukami N, et al. The role of endoscopy in Barrett's esophagus and other premalignant conditions of the esophagus. Gastrointest Endosc. 2012;76(6):1087-1094. doi:10.1016/j. gie. 2012.08 .004

5. Bennett C, Vakil N, Bergman J, et al. Consensus statements for management of Barrett's dysplasia and early-stage esophageal adenocarcinoma, based on a Delphi process. Gastroenterology. 2012;143 (2):336-346. doi:10.1053/j.gastro.2012.04.032

6. Hvid-Jensen F, Pedersen L, Drewes AM, Sørensen HT, Funch-Jensen P. Incidence of adenocarcinoma among patients with Barrett's esophagus. $N$ Engl $J$ Med. 2011;365(15):1375-1383. doi:10.1056/ NEJMoa1103042

7. Shaheen NJ, Falk GW, Iyer PG, Gerson LB. ACG clinical guideline: diagnosis and management of Barrett's esophagus. $\mathrm{Am}$ $J$ Gastroenterol. 2015;111:30. doi:10.1038/ajg.2015.322

8. Gill RS, Singh R. Endoscopic imaging in Barrett's esophagus: current practice and future applications. Ann Gastroenterol. 2012;25(2):89-95.

9. Espino A, Cirocco M, Dacosta R, Marcon N. Advanced imaging technologies for the detection of dysplasia and early cancer in Barrett esophagus. Clin Endosc. 2014;47(1):47-54. doi:10.5946/ ce.2014.47.1.47 
10. Qumseya BJ, Wang H, Badie N, et al. Advanced imaging technologies increase detection of dysplasia and neoplasia in patients with Barrett's esophagus: a meta-analysis and systematic review. Clin Gastroenterol Hepatol. 2013;11(12):1562-1570.e1702. doi:10.1016/ j.cgh.2013.06.017

11. Uno G, Ishimura N, Tada Y, et al. Simplified classification of capillary pattern in Barrett esophagus using magnifying endoscopy with narrow band imaging: implications for malignant potential and interobserver agreement. Medicine. 2015;94(3):e405-e405. doi:10.1097/ MD.0000000000000405

12. Anaparthy R, Sharma P. Progression of Barrett oesophagus: role of endoscopic and histological predictors. Nat Rev Gastroenterol Hepatol. 2014;11(9):525. doi:10.1038/nrgastro.2014.69

13. Kastelein F, van Olphen SH, Steyerberg EW, Spaander MCW, Bruno MJ. Impact of surveillance for Barrett's oesophagus on tumour stage and survival of patients with neoplastic progression. Gut. 2016;65(4):548-554. doi:10.1136/gutjnl-2014-308802

14. Konda VJA, Ross AS, Ferguson MK, et al. Is the risk of concomitant invasive esophageal cancer in high-grade dysplasia in Barrett's esophagus overestimated? Clin Gastroenterol Hepatol. 2008;6 (2):159-164. doi:10.1016/j.cgh.2007.09.013

15. Sharma P, Hawes RH, Bansal A, et al. Standard endoscopy with random biopsies versus narrow band imaging targeted biopsies in Barrett's oesophagus: a prospective, international, randomised controlled trial. Gut. 2013;62(1):15-21. doi:10.1136/gutjnl-2011-300962

16. Sharma P. Clinical practice. Barrett's esophagus. $N$ Engl J Med. 2009;361(26):2548-2556. doi:10.1056/NEJMcp0902173

17. Mansour NM, El-Serag HB, Anandasabapathy S. Barrett's esophagus: best practices for treatment and posttreatment surveillance. Ann Cardiothorac Surg. 2017;6(2):75-87. doi:10.21037/acs.2017.03.05

18. Li H, Hou X, Lin R, et al. Advanced endoscopic methods in gastrointestinal diseases: a systematic review. Quant Imaging Med Surg. 2019;9(5):905-920. doi:10.21037/qims.2019.05.16

19. Sharma P, Bansal A, Mathur S, et al. The utility of a novel narrow band imaging endoscopy system in patients with Barrett's esophagus. Gastrointest Endosc. 2006;64(2):167-175. doi:10.1016/j. gie.2005.10.044

20. Kara MA, Ennahachi M, Fockens P, Ten Kate FJW, Bergman JJGHM. Detection and classification of the mucosal and vascular patterns (mucosal morphology) in Barrett's esophagus by using narrow band imaging. Gastrointest Endosc. 2006;64 (2):155-166. doi:10.1016/j.gie.2005.11.049

21. Anagnostopoulos GK, Yao K, Kaye P, Hawkey CJ, Ragunath K. Novel endoscopic observation in Barrett's oesophagus using high resolution magnification endoscopy and narrow band imaging. Aliment Pharmacol Ther. 2007;26(3):501-507. doi:10.1111/j.13652036.2007.03374.x
22. Singh R, Anagnostopoulos GK, Yao K, et al. Narrow-band imaging with magnification in Barrett's esophagus: validation of a simplified grading system of mucosal morphology patterns against histology. Endoscopy. 2008;40(6):457. doi:10.1055/s-2007-995741

23. Sharma P, Bergman JJGHM, Goda K, et al. Development and validation of a classification system to identify high-grade dysplasia and esophageal adenocarcinoma in Barrett's esophagus using narrowband imaging. Gastroenterology. 2016;150(3):591-598. doi:10.1053/j.gastro.2015.11.037

24. Silva FB, Dinis-Ribeiro M, Vieth M, et al. Endoscopic assessment and grading of Barrett's esophagus using magnification endoscopy and narrow-band imaging: accuracy and interobserver agreement of different classification systems (with videos). Gastrointest Endosc. 2011;73(1):7-14. doi:10.1016/j.gie.2010.09.023

25. Baldaque-Silva F, Marques M, Lunet N, et al. Endoscopic assessment and grading of Barrett's esophagus using magnification endoscopy and narrow band imaging: impact of structured learning and experience on the accuracy of the Amsterdam classification system. Scand J Gastroenterol. 2013;48(2):160-167. doi:10.3109/ 00365521.2012 .746392

26. Muto M, Yao K, Kaise M, et al. Magnifying endoscopy simple diagnostic algorithm for early gastric cancer (MESDA-G). Dig Endosc. 2016;28(4):379-393. doi:10.1111/den.12638

27. Hayee B, Inoue H, Sato H, et al. Magnification narrow-band imaging for the diagnosis of early gastric cancer: a review of the Japanese literature for the Western endoscopist. Gastrointest Endosc. 2013;78 (3):452-461. doi:10.1016/j.gie.2013.03.1333

28. Yao K, Anagnostopoulos GK, Ragunath K. Magnifying endoscopy for diagnosing and delineating early gastric cancer. Endoscopy. 2009;41(5):462-467. doi:10.1055/s-0029-1214594

29. Montgomery E, Bronner MP, Goldblum JR, et al. Reproducibility of the diagnosis of dysplasia in Barrett esophagus: a reaffirmation. Hum Pathol. 2001;32(4):368-378. doi:10.1053/hupa.2001.23510

30. Vennalaganti P, Kanakadandi V, Goldblum JR, et al. Discordance among pathologists in the United States and Europe in diagnosis of low-grade dysplasia for patients with Barrett's esophagus. Gastroenterology. 2017;152(3):564-570.e564. doi:10.1053/j. gastro.2016.10.041

31. Thosani N, Abu Dayyeh BK, Sharma P, et al. ASGE technology committee systematic review and meta-analysis assessing the ASGE preservation and incorporation of valuable endoscopic innovations thresholds for adopting real-time imaging-assisted endoscopic targeted biopsy during endoscopic surveillance of Barrett's esophagus. Gastrointest Endosc. 2016;83(4):684-698.e687. doi:10.1016/j. gie.2016.01.007
Clinical and Experimental Gastroenterology

\section{Publish your work in this journal}

Clinical and Experimental Gastroenterology is an international, peerreviewed, open access, online journal publishing original research, reports, editorials, reviews and commentaries on all aspects of gastroenterology in the clinic and laboratory. This journal is indexed on American Chemical Society's Chemical Abstracts Service (CAS).
The manuscript management system is completely online and includes a very quick and fair peer-review system, which is all easy to use. Visit http://www.dovepress.com/testimonials.php to read real quotes from published authors. 